\title{
RESEARCH
}

Open Access

\section{Systemic TLR2 tolerance enhances central nervous system remyelination}

Nicholas J. Wasko', Meghan Horne Kulak', Debayon Paul', Alexandra M. Nicaise ${ }^{3}$, Stephen T. Yeung ${ }^{1,5}$, Frank C. Nichols ${ }^{4}$, Kamal M. Khanna ${ }^{1,5}$, Stephen Crocker $^{3}$, Joel S. Pachter ${ }^{1}$ and Robert B. Clark ${ }^{1,2^{*}}$ (D)

\begin{abstract}
Background: Multiple sclerosis (MS) is a central nervous system (CNS) autoimmune disease characterized by both inflammatory demyelination and impaired remyelination. Studies indicate that Toll-like receptor 2 (TLR2) signaling contributes to both the inflammatory component and the defective remyelination in MS. While most MS therapeutics target adaptive immunity, we recently reported that reducing TLR2 signaling in innate immune cells by inducing TLR2 tolerance attenuates adoptively transferred experimental autoimmune encephalomyelitis. Given that previous reports suggest TLR2 signaling also inhibits myelin repair, the objective of this study was to assess how reducing TLR2 signaling through TLR2 tolerance induction affects CNS myelin repair.

Methods: Chow containing 0.2\% cuprizone was fed to male and female wild-type (WT) C57BL/6 mice or TLR2-deficient $\left(\mathrm{TLR}^{-{ }^{-}}\right)$mice for 5 weeks to induce demyelination. During a 2-week remyelination period following discontinuation of cuprizone, WT mice received either low dose TLR2 ligands to induce systemic TLR2 tolerance or vehicle control (VC). Remyelination was evaluated via electron microscopy and immunohistochemical analysis of microglia and oligodendrocytes in the corpus callosum. Statistical tests included 2-way ANOVA and Mann-Whitney $U$ analyses.
\end{abstract}

Results: Inducing TLR2 tolerance in WT mice during remyelination significantly enhanced myelin recovery, restoring unmyelinated axon frequency and myelin thickness to baseline levels compared to VC-treated mice. Mechanistically, enhanced remyelination in TLR2 tolerized mice was associated with a shift in corpus callosum microglia from a pro-inflammatory iNOS ${ }^{+}$phenotype to a non-inflammatory/pro-repair $\mathrm{Arg}^{+}$phenotype. This result was confirmed in vitro by inducing TLR2 tolerance in WT microglia cultures. TLR2 ${ }^{-l-}$ mice, without TLR2 tolerance induction, also significantly enhanced myelin recovery compared to WT mice, adding confirmation that reduced TLR2 signaling is associated with enhanced remyelination.

Discussion: Our results suggest that reducing TLR2 signaling in vivo by inducing TLR2 tolerance significantly enhances myelin repair. Furthermore, the enhanced remyelination resulting from TLR2 tolerance induction is associated with a shift in corpus callosum microglia from a pro-inflammatory iNOS ${ }^{+}$phenotype to a non-inflammatory/ pro-repair Arg $1^{+}$phenotype. While deletion of TLR2 would be an impractical approach in vivo, reducing innate immune signaling through TLR2 tolerance induction may represent a novel, two-pronged approach for treating both inflammatory and myelin repair components of MS.

Keywords: TLR, TLR2, Tolerance, Remyelination, Multiple sclerosis, Microglia

\footnotetext{
* Correspondence: rclark@uchc.edu

${ }^{1}$ Department of Immunology, UConn Health, Farmington, CT 06030, USA

2Department of Medicine, UConn Health, Farmington, CT 06030, USA

Full list of author information is available at the end of the article
}

(c) The Author(s). 2019 Open Access This article is distributed under the terms of the Creative Commons Attribution 4.0 International License (http://creativecommons.org/licenses/by/4.0/), which permits unrestricted use, distribution, and reproduction in any medium, provided you give appropriate credit to the original author(s) and the source, provide a link to the Creative Commons license, and indicate if changes were made. The Creative Commons Public Domain Dedication waiver (http://creativecommons.org/publicdomain/zero/1.0/) applies to the data made available in this article, unless otherwise stated. 


\section{Introduction}

Multiple sclerosis (MS) is a progressive demyelinating disease of the central nervous system (CNS) characterized by immune-mediated damage to the myelin sheath surrounding neuronal axons [1]. Approximately $80 \%$ of cases present as relapsing-remitting MS (RRMS), with periodic clinical attacks punctuating periods of stability. Many such cases transition into secondary progressive MS (SPMS), in which neurological disability accumulates more consistently [1]. Alternatively, primary progressive MS (PPMS) occurs in 15\% of cases, manifesting as continuous deterioration of neural function [2]. Immunetargeting treatments can reduce symptomatic flares in RRMS, but no existing therapies demonstrate consistent efficacy in treating SPMS or PPMS [3, 4]. The pathophysiology of MS includes loss of myelin-producing oligodendrocytes (OLs) [5], and while demyelination can theoretically be repaired by differentiation of oligodendrocyte precursor cells (OPCs) into mature OLs, for reasons as yet unknown, remyelination is defective in MS [5-8]. Diminished differentiation of OPCs into mature OLs may contribute to this defect in remyelination [3, 8, 9]. Enhancing remyelination has become an increasingly important goal of MS research.

Although the role of the adaptive immune system is well established in both MS and its murine model, experimental autoimmune encephalomyelitis (EAE), the role of the innate immune system has been less well explored. Studies have suggested that signaling through Toll-like receptor 2 (TLR2) plays a critical role in the inflammatory pathogenesis of MS and EAE [10-12], but only few studies have investigated a role for TLR2 signaling in remyelination. Of these, most have demonstrated an inhibitory effect of TLR2 signaling on remyelination [13-15].

Identifying the role of TLR2 signaling in the pathogenesis of MS is complicated by a potential role for the microbiome. One postulated function of the microbiome is to set the homeostatic threshold for TLR2 responsiveness through a relative "tolerance induction," or regulation mediated by seeding the systemic circulation with low levels of TLR2 signaling microbial products $[10,16]$. In proofof-concept studies, we demonstrated that: a microbiomederived bacterial lipopeptide, Lipid 654, can be detected in the serum of all healthy individuals but is found at significantly lower levels in the serum of MS patients [16]; induction of TLR2 tolerance via administration of low-dose TLR2 ligands results in the attenuation of adoptive transfer murine EAE [10]; and a significant proportion of MS patients demonstrate enhanced responsiveness to TLR2 stimulation [17].

Based on studies relating TLR2 to the inhibition of remyelination [13-15] and the potential for therapeutic intervention in EAE and MS via TLR2 tolerance induction [10, 17], the goal of the present study was to test the role of TLR2 and TLR2 tolerance induction in the process of CNS remyelination. Using the cuprizone model of demyelination, we now report that remyelination is enhanced when TLR2 signaling is diminished by the induction of TLR2 tolerance. These findings thus raise the possibility that this approach might ameliorate both the inflammatory and remyelinating aspects of MS pathogenesis.

\section{Methods \\ Mice}

Male and female C57BL/6 wild-type (WT) mice were purchased from Jackson Laboratory (Bar Harbor, ME). $\mathrm{TLR}^{-/-}$mice, bred onto a C57BL/6 background, were a generous gift of S. Akira (Osaka University, Japan). All mice were maintained under specific pathogen-free conditions in accordance with the guidelines for the Center for Comparative Medicine at UConn Health. All procedures were performed in compliance with Institutional Animal Care and Use Committee-approved protocols.

\section{Cuprizone demyelination model}

Male or female wild-type (WT) C57BL/6 mice or $\mathrm{TLR}^{-/-}$mice (6-10 weeks old) were fed milled chow containing $0.2 \%$ bis (cyclohexanone) oxaldihydrazone (cuprizone; Sigma-Aldrich, St. Louis, MO), with food and water available ad libitum. Cuprizone feeding was maintained for 35 days to induce demyelination, then changed to normal rodent chow for the following 14 days to allow remyelination to occur.

\section{In vivo TLR2 tolerance induction}

Starting on day 33, mice received intravenous (i.v.) injections of PBS vehicle control (VC) or $\mathrm{Pam}_{2} \mathrm{CSK}_{4}$ (P2C) $(2.5 \mu \mathrm{g})$ (InvivoGen; San Diego, CA). Injections were given once per day for 5 days, then every other day through the remainder of the 14-day post-cuprizone recovery period.

\section{Assessment of in vivo TLR2 tolerance}

To test for systemic TLR2 tolerance, on day 14 of the post-cuprizone recovery period, mice received an i.v. injection of $100 \mu \mathrm{g} \mathrm{Pam} 3 \mathrm{CSK}_{4}$ (P3C; InvivoGen). Mice were bled $2 \mathrm{~h}$ later, and serum TNF $\alpha$ was assayed using ReadySET-Go! ELISA kits (Affymetrix; Santa Clara, CA).

\section{Electron microscopy}

On day 15 of the post-cuprizone recovery period, mice were anesthetized with ketamine and perfused transcardially with $5 \mathrm{~mL}$ of PBS followed by $10 \mathrm{~mL}$ of cold $2 \%$ paraformaldehyde (PFA) $/ 2.5 \%$ glutaraldehyde. Brains were removed, stored overnight at $4{ }^{\circ} \mathrm{C}$ in $2 \% \mathrm{PFA} / 2.5 \%$ glutaraldehyde, then the corpus callosum (CC) was 
dissected out and transferred to $0.1 \mathrm{M}$ cacodylate buffer. This tissue was then post-fixed in $\mathrm{OsO}_{4}$, dehydrated in ethanol, and embedded in epoxy resin. Ultrathin 70-nm sections were stained with uranyl acetate and Sato's Lead citrate and examined with a Hitachi H-7650 TEM.

\section{Assessment of myelination}

Digitized, non-overlapping electron micrographs of the $\mathrm{CC}$ were analyzed for unmyelinated axon frequency and g-ratios. To calculate the unmyelinated axon frequency, $300+$ axons from representative images $\left(500 \mu \mathrm{m}^{2}\right.$ per animal) were analyzed. Unmyelinated axons were counted as those with a total absence of surrounding myelin. For gratio analysis, a minimum of 100 randomly selected axons were measured using a plug-in for the ImageJ software which allowed for semi-automated analysis of randomly selected sets of fibers [18]. Fibers with prominent outfoldings in the plane of section were excluded.

\section{Tissue section preparation}

On day 15 of the post-cuprizone recovery period, mice were anesthetized with ketamine and perfused transcardially with $5 \mathrm{~mL}$ of PBS followed by $10 \mathrm{~mL}$ of cold $4 \%$ PFA. Brains were removed, post-fixed overnight in $4 \%$ PFA, then cryoprotected in 30\% sucrose. Coronal sections of $40 \mu \mathrm{m}$ thickness (Bregma $+1.10 \mathrm{~mm}$ to -2.30 $\mathrm{mm}$ ) were cut using a Microm HM440E microtome. Sections were stored at $4{ }^{\circ} \mathrm{C}$ in PBS containing $0.05 \%$ sodium azide until stained.

\section{Immunohistofluorescence}

Tissue sections were blocked and permeabilized at room temperature for $1 \mathrm{~h}$ in $5 \%$ bovine serum albumin (Sigma), $0.05 \%$ Triton X-100 (Sigma), and 5\% heat-inactivated donkey serum (Millipore) or goat serum (Millipore) for microglia or $\mathrm{OPC} / \mathrm{OL}$ staining, respectively. Antibodies for microglia stains were 1:1,000 rabbit anti-IBA1 (Wako; Richmond, VA), 1:200 mouse anti-iNOS (Invitrogen; San Diego, CA), 1:50 goat anti-Arg1 (NovusBio; Littleton, CO), 1:500 donkey anti-rabbit IgG Alexafluor 546 (Invitrogen), 1: 500 donkey anti-mouse IgG Alexa-Fluor 647 (Invitrogen), and 1:250 donkey anti-goat IgG Alexa-Fluor 488 (Jackson Labs). Antibodies for OPC/OL stains were 1:250 rabbit anti-Olig2 (NovusBio), 1:500 mouse anti-APC, clone CC1 (Millipore), 1:500 rat anti-PDGFR $\alpha$ (Invitrogen), 1:500 goat anti-rabbit IgG Alexa-Fluor 647 (Invitrogen), 1:1,000 goat anti-mouse IgG Alexa-Fluor 546 (Invitrogen), and 1:2,000 goat anti-rat IgG Alexa-Fluor 488 (Abcam; Cambridge, MA). Primary antibodies were incubated overnight at $4{ }^{\circ} \mathrm{C}$ and secondary antibodies were incubated for $2 \mathrm{~h}$ at room temperature. Nuclei were stained with 1:1,000 DAPI (Thermo Fisher Scientific; Waltham, MA) for $10 \mathrm{~min}$ at room temperature. Slides were mounted with Mowiol (Sigma), imaged with a Zeiss LSM 880 confocal microscope, acquired using Zen Black software, and analyzed on Bitplane IMARIS software (Concord, MA).

\section{In vitro microglia TLR2 tolerance}

Whole brain cultures were established from P1-P3 WT mouse pups and plated in T75 flasks as described previously [19]. The culture media was removed and replaced twice per week for approximately $10-15$ days. When adherent astrocytes were confluent for at least 5 days, nonadherent cells were harvested and assessed for microglial purity by flow cytometry after labeling with antibodies to CD45.2 and CD11b. In 4 of 6 studies, non-adherent cells were further purified by FACS-sorting for $\mathrm{CD} 45.2^{+} \mathrm{CD} 11 \mathrm{~b}^{+}$ microglia (Additional file 1: Fig. S1). Non-adherent cells (65-75\% CD $45.2^{+}$CD11b ${ }^{+}$), or FACS-sorted microglia (> $\left.99 \% \mathrm{CD} 45.2^{+} \mathrm{CD} 11 \mathrm{~b}^{+}\right)$, were plated at $2-7 \times 10^{4}$ cells/well in 96-well plates pre-coated with poly-L Lysine $(0.1 \mathrm{mg} /$ $\mathrm{mL})$. Ninety-six-well microglial cultures were designated as either "non-stimulated," "stimulated," or "tolerized." "Nonstimulated" wells received no stimulation over the ensuing entire 2 days of culture. "Stimulated" wells received $1 \mu \mathrm{g} /$ $\mathrm{mL} \mathrm{P2C} \mathrm{at} \mathrm{the} 24$ hour time point. "Tolerized" wells received $1 \mu \mathrm{g} / \mathrm{mL}$ P2C both at the initiation of culture and at the 24 hour time point. After 48 hours, supernatants were harvested and assayed for cytokines via multiplex enzymelinked immunosorbent assay (ELISA).

\section{Statistical analysis}

Figures 1c, 2, 3, and 4 utilized 2-way ANOVA, with values below $p=0.05$ considered significant. Figures 5,6 , and 7 utilized Mann-Whitney $U$ analyses, with values below $p=0.05$ considered significant.

\section{Results}

\section{In vivo induction of TLR2 tolerance}

To assess the effect of TLR2 tolerance on myelin repair, we utilized the cuprizone model of demyelination. WT mice were fed chow containing cuprizone for 5 weeks to induce demyelination, then given a 2-week remyelination ("recovery") period during which time they were fed normal chow (Fig. 1a). Alternatively, WT mice received normal chow for the entire 7 week period. Remyelination was then assessed in the $\mathrm{CC}$ of all cohorts at the end of the 2-week recovery period.

It is well documented both in vitro and in vivo that stimulation with a TLR ligand can induce significant downregulation of pro-inflammatory responses upon subsequent TLR ligation ("tolerance induction") [20]. In this case, TLR2 tolerance was induced in the 5-week cuprizone/2-week normal chow cohort as well as in the 7 -week normal chow cohort, starting on the last 2 days of week 5 and continuing through the 2-week "recovery" period. TLR2 tolerance was induced by repeated administration of a low dose of the TLR2 ligand $\mathrm{Pam}_{2} \mathrm{CSK}_{4}$ 


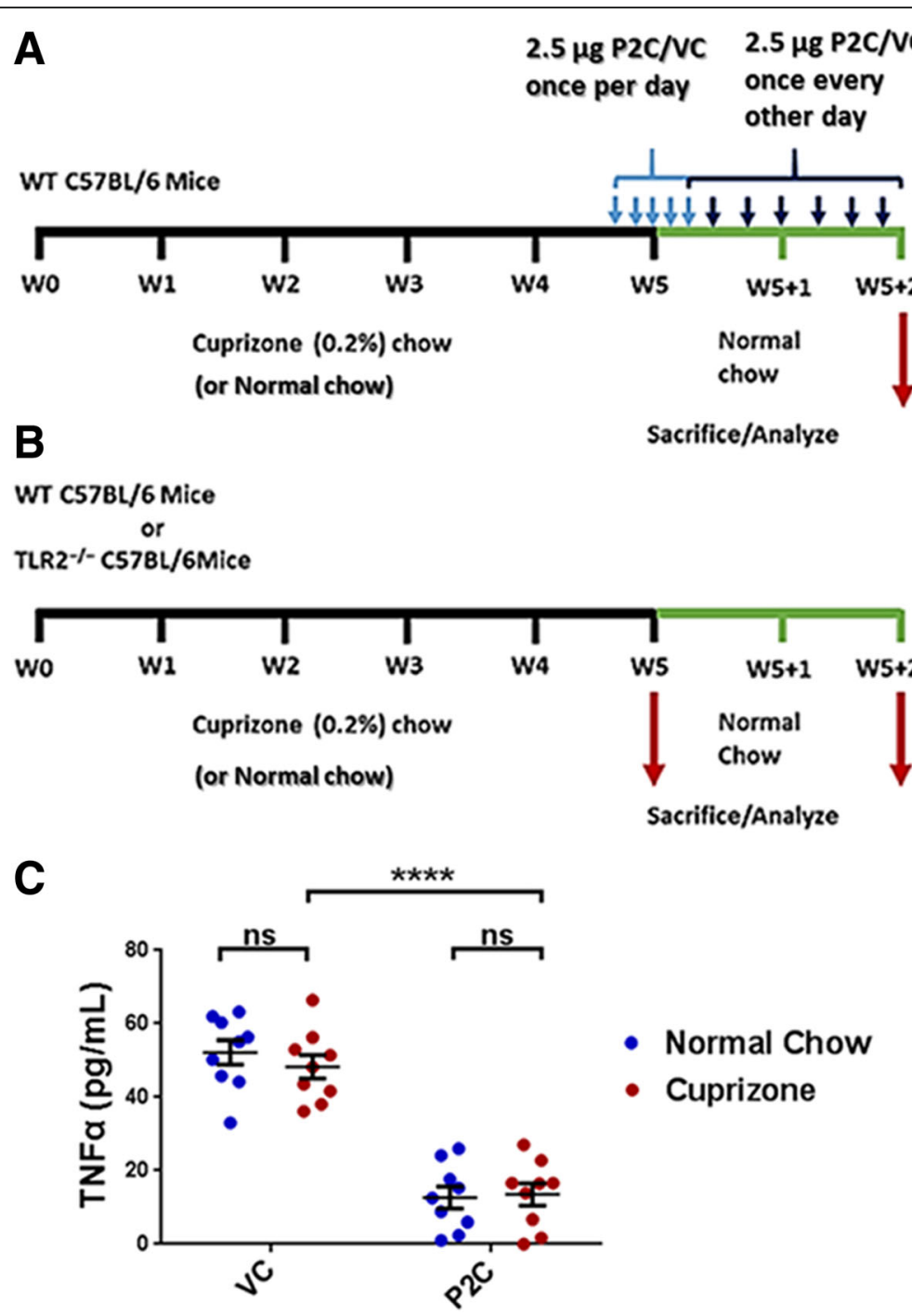

Fig. 1 a Timeline of TLR2 tolerance induction. Timeline for the 5-week induction of demyelination with $0.2 \%$ cuprizone (or normal chow), followed by the 2-week remyelination period on normal chow. WT mice were treated with either low dose P2C or VC during the remyelination period. Mice were sacrificed and analyzed after the 2-week remyelination period. $\mathbf{b}$ Timeline of TLR2 ${ }^{-1-}$. Timeline for the 5 -week induction of demyelination with $0.2 \%$ cuprizone (or normal chow) followed by the 2-week remyelination period. WT and TLR2 ${ }^{-/-}$mice were not treated during the remyelination period. Mice were sacrificed and analyzed immediately following the 5 -week cuprizone (or normal chow) feeding to evaluate baseline demyelination levels, or after the 2-week remyelination period on normal chow to evaluate myelin recovery. c Systemic TLR2 tolerance induction. At the end of the 2-week remyelination period, WT mice that had been fed for the first 5 weeks with either cuprizone or normal chow and treated with P2C or VC as in a received a single i.v. injection of Pam3CSK 4 (P3C, $100 \mu \mathrm{gg})$ and $2 \mathrm{~h}$ later serum was obtained and analyzed for TNFa by ELISA. Results are expressed as mean values per mouse. $N=9$ mice/cohort. Error bars represent the mean \pm SEM. Statistical differences were assessed by 2 -way ANOVA. ${ }^{* * *} p<0.0001$. For NS comparisons, VC treated: normal vs cuprizone-fed, $p=0.8118$. P2C treated: normal vs cuprizone-fed, $p=0.9972$

(P2C); $2.5 \mu \mathrm{g}$ of P2C was given i.v. once per day for 5 days, then every other day for the remaining 12 days of the 14-day recovery period. Control cohorts of mice on the 5-week cuprizone/2-week normal chow schedule, as well as mice on the 7-week normal chow schedule, received vehicle control (VC) i.v. rather than P2C (Fig. 1a).

At the end of the 2-week recovery period, systemic TLR2 tolerance was assessed by challenging mice with a single i.v. injection of a large dose $(100 \mu \mathrm{g})$ of a different
TLR2 ligand, Pam3CSK 4 (P3C). The systemic response to the P3C challenge was analyzed by obtaining serum samples $2 \mathrm{~h}$ later and measuring serum TNF $\alpha$ levels by ELISA. Mice treated with VC, regardless of whether they had been on the 5-week cuprizone/2 week normal chow schedule or the 7-week normal chow schedule, showed a strong systemic response to the $\mathrm{P} 3 \mathrm{C}$ challenge as indicated by high levels of serum TNF $\alpha$ (Fig. 1c). In contrast, mice treated with $\mathrm{P} 2 \mathrm{C}$ throughout the recovery 

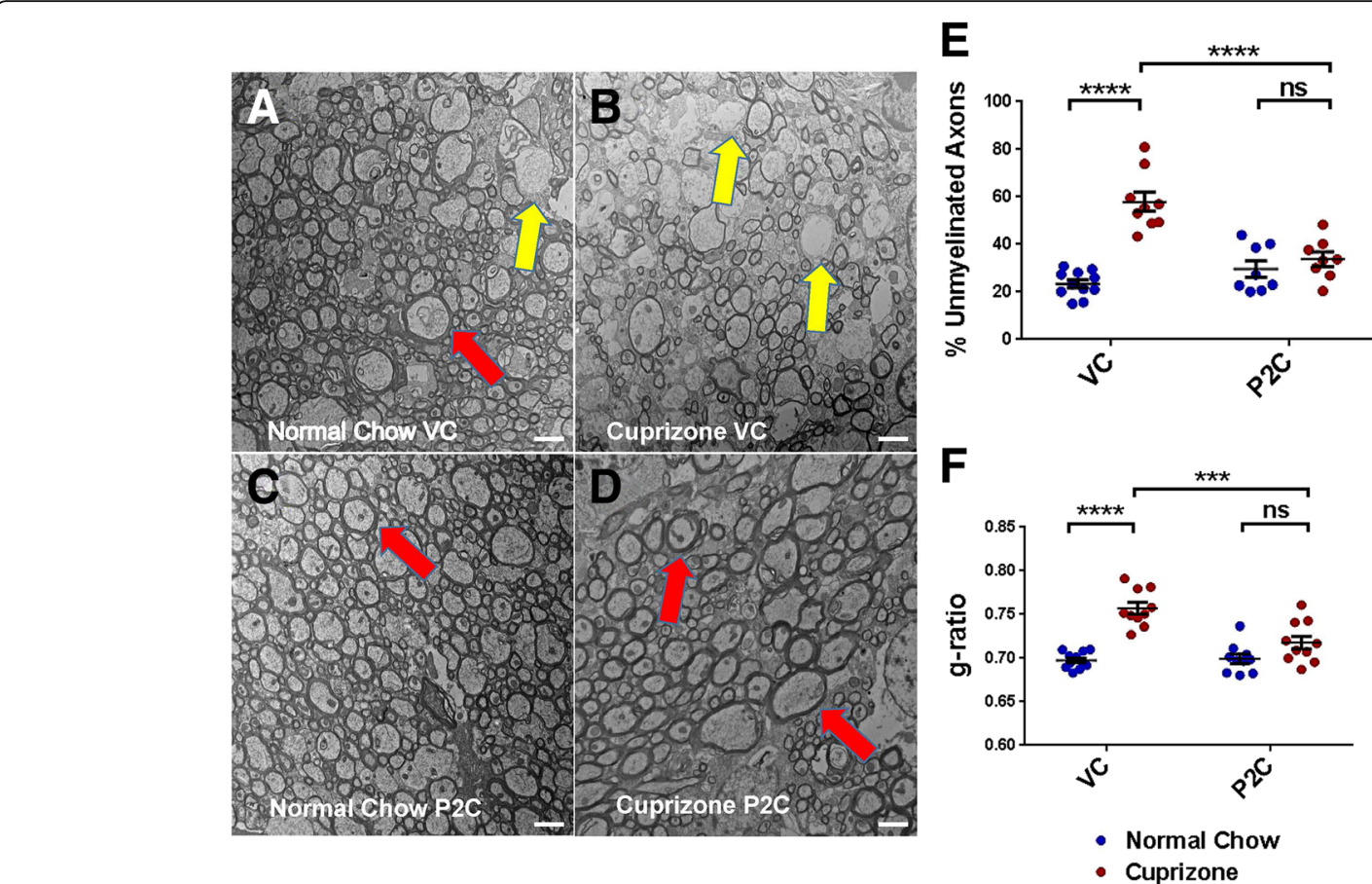

Fig. 2 Effect of TLR2 tolerance induction on remyelination. a-d Representative EM images from corpus callosum: a "Normal Chow VC" - mice fed for 7 weeks with normal chow and treated for the last 2 weeks with VC. b "Cuprizone VC"-mice fed for 5 weeks with cuprizone, then fed for 2 weeks with normal chow and treated for these last 2 weeks with VC. c "Normal Chow P2C"-mice fed for 7 weeks with normal chow and treated for the last 2 weeks with Pam2CSK 4 (P2C). $\mathbf{d}$ "Cuprizone P2C"-mice fed for 5 weeks with cuprizone, then fed for 2 weeks with normal chow and treated for these last 2 weeks with P2C. Scale bar $=2 \mu \mathrm{m}$. Red arrows depict typical axons with normal myelin thickness. Yellow arrows depict typical axons with decreased myelin thickness. e Percentage of unmyelinated axons as calculated from EM images. $\mathbf{f}$ Myelin thickness ( $\mathrm{g}$-ratios) as calculated from EM images. For $\mathbf{e}$ and $\mathbf{f}$, data points represent individual mice analyzed as described in the "Methods" section. $N=8-10$ mice per experimental condition. Error bars represent the mean \pm SEM. Statistical differences were assessed by 2-way ANOVA. ${ }^{* * *} p<0.001 ;{ }^{* * * *} p<0.0001$. For NS comparisons: P2C treated: normal vs cuprizone-fed, $p=0.7912$ (e). P2C treated: normal vs cuprizone-fed, $p=0.1486$ (f)

period, regardless of prior feeding schedule, demonstrated a significantly reduced systemic TNF $\alpha$ response to the P3C challenge (Fig. 1c). These results indicate that repeated low-dose $\mathrm{P} 2 \mathrm{C}$ injections during the recovery period induced a state of systemic TLR2 tolerance, with a significant decrease in the ligand-activated systemic TLR2 pro-inflammatory response.

\section{TLR2 tolerance promotes remyelination following cuprizone-induced demyelination}

To assess the efficiency of remyelination following 5 weeks of cuprizone and 2 weeks of recovery, mice were sacrificed and myelination in the $\mathrm{CC}$ analyzed by electron microscopy (EM). Electron micrographs of CC cross sections were evaluated for the percentage of myelinated fibers and thickness of myelin sheaths (i.e., g-ratio). Among the VCtreated mice, those fed with cuprizone demonstrated a significantly higher percentage of axons lacking myelin ("unmyelinated") compared to those fed with normal chow for the first 5 weeks (Fig. 2a, b, e). In contrast, among the P2C treated/TLR2-tolerized mice, those fed with cuprizone showed no significant increase in the percentage of unmyelinated axons compared to those fed with normal chow
(Fig. 2c-e). These results suggest that inducing TLR2 tolerance during the recovery period restored the frequency of unmyelinated axons to baseline levels (Fig. 2e).

In addition to measuring the percentage of myelinated fibers, myelin thickness was measured using EM analysis of g-ratios. G-ratios were calculated as the ratio of the inner axonal diameter to the total outer diameter. Among VC-treated mice, those fed with cuprizone demonstrated a significantly higher g-ratio (i.e., less myelin thickness) than those fed with normal chow (Fig. 2a, b, f). In contrast, among P2C/TLR2-tolerized mice, those fed with cuprizone showed no significant increase in g-ratio (i.e., no thinning of myelin) compared to those fed with normal chow (Fig. 2c, d, f). These data suggest that inducing TLR2 tolerance during the recovery period restored the myelin thickness to baseline levels (Fig. 2f).

\section{TLR2 tolerance does not increase OPC or OL frequency following cuprizone-induced demyelination}

It has been reported that OPCs express TLR2 and that ligation of TLR2 can have an inhibitory effect on OPC maturation to mature OLs [15]. To determine whether the improved rate of myelin repair found in P2C-treated/ 

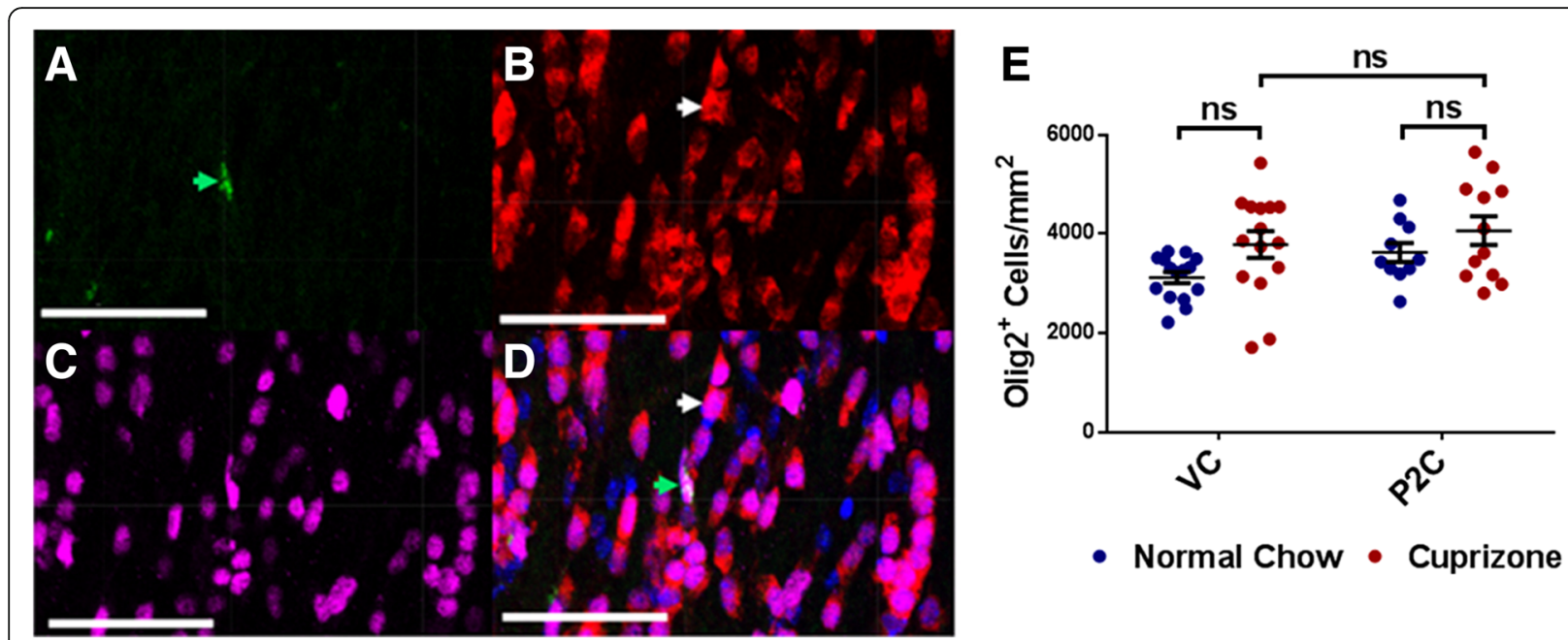

- Normal Chow • Cuprizone
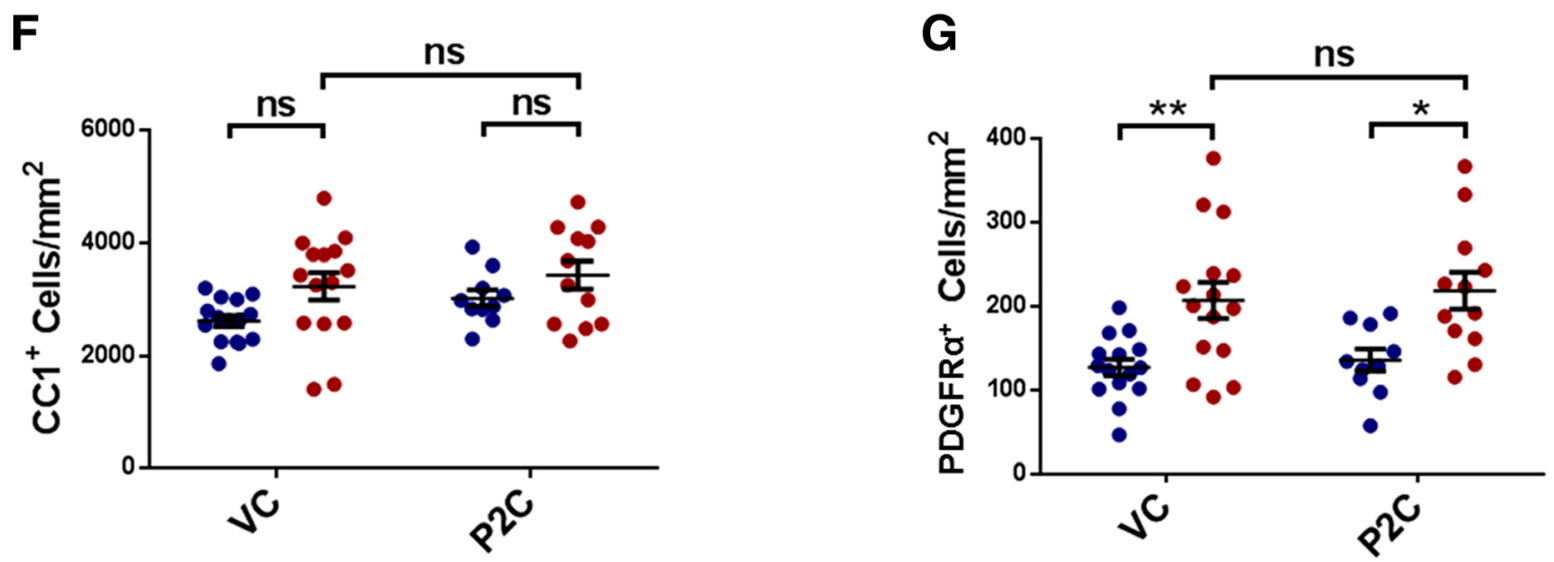

Fig. 3 Effect of TLR2 tolerance induction on OPC and OL frequency. a-d Overlapping immunohistofluorescent stains of one representative corpus callosum section. $\mathbf{a}$ Green $=$ PDGFRa ${ }^{+}$cells, $\mathbf{b}$ red $=\mathrm{CC}^{+}$cells, $\mathbf{c}$ magenta $=$ Olig2 ${ }^{+}$cells, $\mathbf{d}$ all images merged; blue $=$ DAPI. In $\mathbf{d}$, the white arrow depicts the example $\mathrm{CC}^{+}$cell also seen in $\mathbf{b}$, and the green arrow depicts the example PDGFRa ${ }^{+}$cell also seen in $\mathbf{a}$. Scale bars represent 50 um. e-g Analysis of cell frequency in corpus callosum sections based on co-localization of DAPI ${ }^{+} \mathrm{Olig}^{+}$cells $/ \mathrm{mm}^{2}$. e Olig2 $2^{+}$cells $/ \mathrm{mm}^{2}, \mathbf{f}$ $\mathrm{Olig2}^{+} \mathrm{CC}^{+}$cells $/ \mathrm{mm}^{2}, \mathbf{g}$ Olig2 ${ }^{+}$PDGFRa ${ }^{+}$cells $/ \mathrm{mm}^{2}$. Data points represent individually analyzed sections derived from 4 mice per cohort; 3-4 sections were analyzed from each mouse. $N=12-15$ total sections per experimental cohort. Error bars represent the mean \pm SEM. Statistical differences were assessed by 2 -way ANOVA. ${ }^{*} p<0.05 ;{ }^{* *} p<0.01$. For NS comparisons: e VC treated: normal vs cuprizone-fed, $p=0.1660$. P2C treated: normal vs cuprizone-fed, $p=0.7672$. Normal chow: VC Vs P2C, $p=0.5803$. Cuprizone-fed: VC Vs P2C, $p=0.9451$. f VC treated: normal vs cuprizone-fed, $p=0.1295$. P2C treated: normal vs cuprizone-fed, $p=0.7142$. Normal chow: VC vs P2C, $p=0.6692$. Cuprizone-fed: VC vs P2C, $p=$ 0.9772. g Cuprizone-fed: VC vs P2C, $p=0.9983$

TLR2-tolerized mice reflected increased numbers of OPCs or increased maturation of OPCs into myelin-producing OLs, mice treated either with VC or P2C during the 2week recovery period were evaluated using an immunohistofluorescence approach. CC sections were stained with anti-Olig2 antibodies, which delineated all cells of OL lineage, anti-PDGFR $\alpha$ antibodies to label OPCs, or antiAPC (CC1 clone) antibodies to label mature OLs. Representative stains are shown in Fig. 3a-d. We then analyzed the absolute numbers of Olig2 ${ }^{+}$cells, the absolute numbers of Olig2 ${ }^{+} \mathrm{CC}^{+}$mature OL cells, and the absolute numbers of Olig2 ${ }^{+}$PDGFR $^{+}$OPCs.

Although not statistically significant, cuprizone-fed mice exhibited slightly higher numbers of $\mathrm{Olig}^{+}$cells than mice that received only normal chow, which was seen in both VC- and P2C-treated cohorts (Fig. 3e). Absolute numbers of $\mathrm{CC}^{+}$mature OLs showed a similar trend, which also did not differ significantly between VC and P2C-treated cohorts (Fig. 3f). Cuprizone-fed mice did demonstrate a significant increase in PDGFR $\alpha^{+}$ OPCs compared to mice that received normal chow. However, this increase in PDGFR $\alpha^{+}$OPCs was present and equal in both VC and P2C-treated cohorts (Fig. 3g). These results indicate that inducing TLR2 tolerance with P2C did not significantly alter the absolute numbers of OPCs or mature OLs compared to VC-treated mice (Fig. 3f, g). This suggests that the enhancement of remyelination seen in P2C-treated/TLR2-tolerized mice 

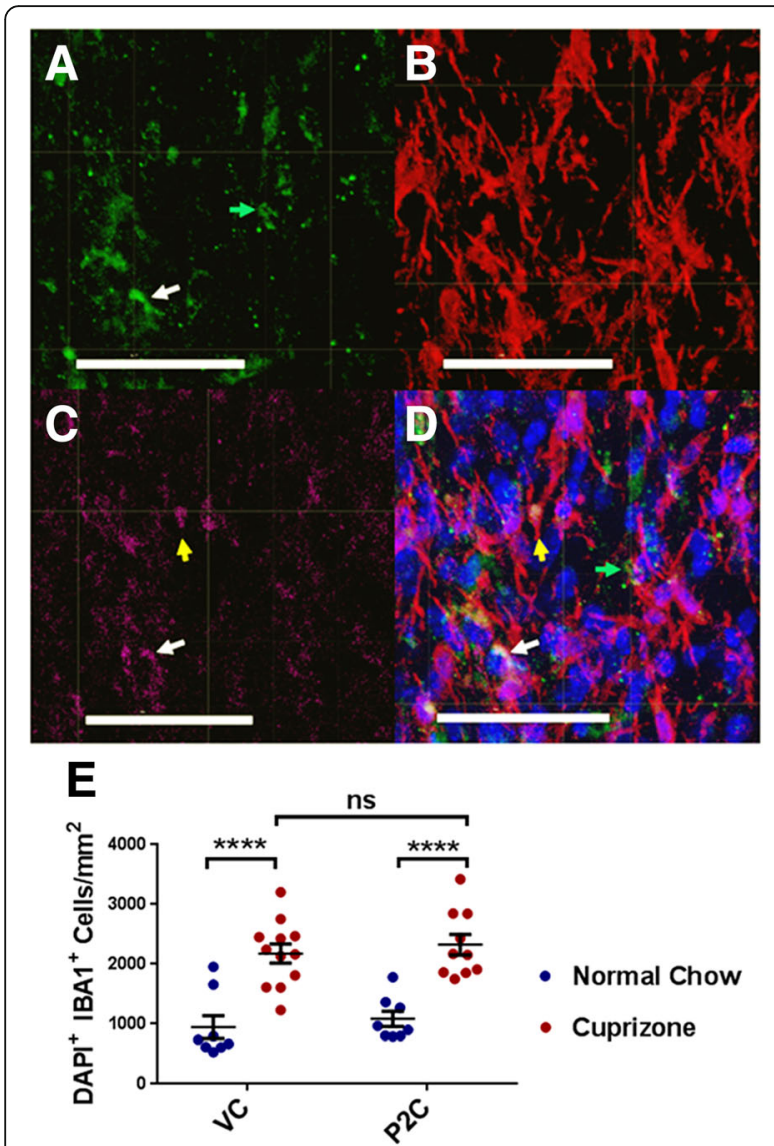
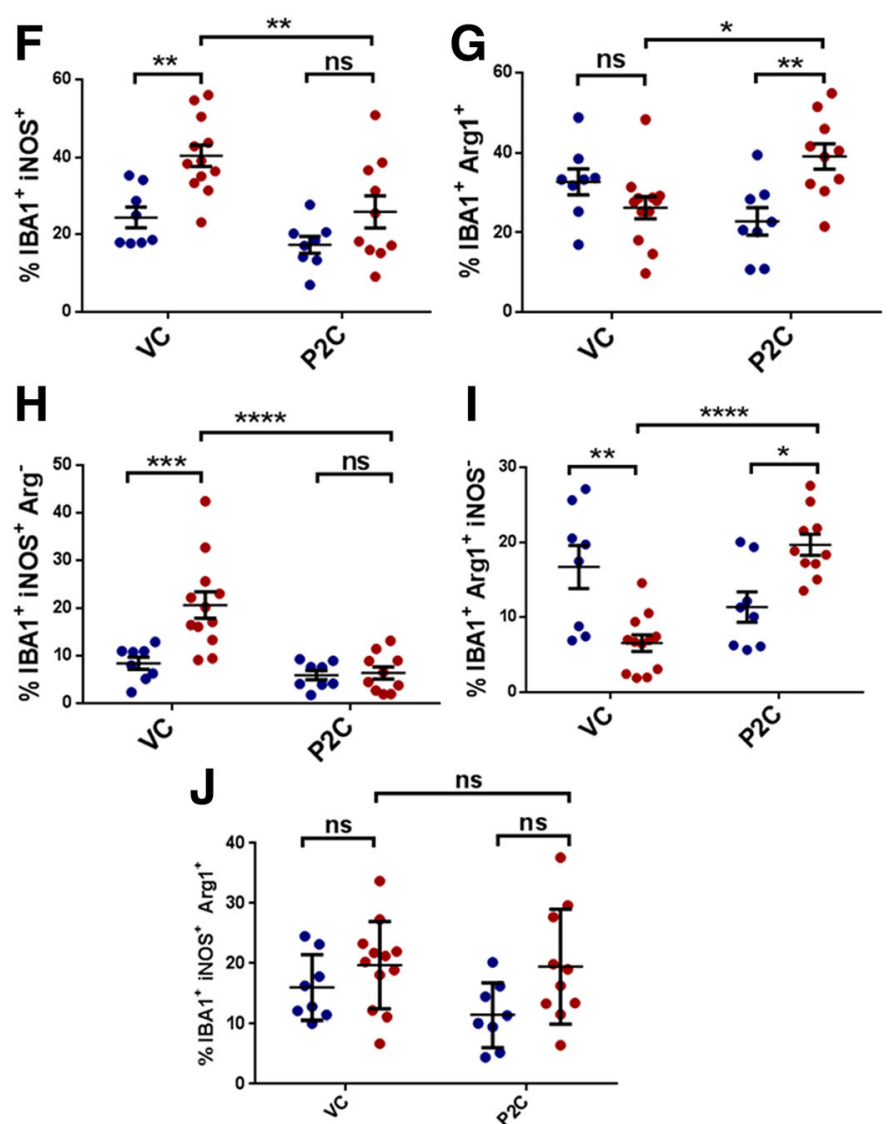

Fig. 4 Effect of TLR2 tolerance induction on microglia frequency and phenotype. a-d overlapping immunohistofluorescent stains of one representative corpus callosum section. a green $=A \mathrm{Arg}^{+}$cells, $\mathbf{b}$ red $=\mid \mathrm{BA} 1^{+}$cells; $\mathbf{c}$ magenta $=\mathrm{iNOS}{ }^{+}$cells; $\mathbf{d}$ all images merged; blue $=\mathrm{DAPI} . \mathrm{In}$ d, the green arrow depicts the example $\mathrm{Arg}^{+}{ }^{+}$cell also shown in $\mathbf{a}$, the yellow arrow depicts the example iNOS ${ }^{+}$cell also seen in $\mathbf{c}$, and the white arrow depicts the example double-positive, $\mathrm{NOSS}^{+} \mathrm{Arg} 1^{+}$cell, also seen in a and $\mathbf{c}$. Scale bars represent $50 \mu \mathrm{m}$. e Analysis of IBA ${ }^{+}$cell frequency in corpus callosum sections, based on co-localization of DAPI $\mid \mathrm{BAA} 1^{+}$cells. $\mathbf{f}$ Percentage of $\mid \mathrm{BA} 1^{+}$cells that are iNOS ${ }^{+}$(total $=$single and double positives). $\mathbf{g}$ Percentage of $\mid \mathrm{BA} 1^{+}$cells that are $\mathrm{Arg}^{+}{ }^{+}$(total $=$single and double positives). $\mathbf{h}$ Percentage of $\mid \mathrm{BA} 1^{+}$cells that are iNOS ${ }^{+}$ single positive $\left(\mathrm{Arg}^{-}{ }^{-}\right)$. $\mathbf{i}$ Percentage of $\mid \mathrm{BA} 1^{+}$cells that are $\mathrm{Arg}^{1^{+}}$single positive (iNOS ${ }^{-}$). $\mathbf{j}$ Percentage of $\mid \mathrm{BA} 1^{+}$cells that are $\mathrm{iNOS}^{+} \mathrm{Arg}^{+}$doublepositive. Data points represent individually analyzed sections derived from 4 mice per cohort; $2-3$ sections were analyzed from each mouse. $N=8-12$ total sections per experimental cohort. Error bars represent the mean \pm SEM. Statistical differences were assessed by 2-way ANOVA, ${ }^{*} p<0.05$; ${ }^{* *} p<0.01$; ${ }^{* *} p<0.001$; ${ }^{* * *} p<0.0001$. For NS comparisons: e Cuprizone-fed: VC vs P2C, $p=0.9042$. f P2C treated: normal vs cuprizone-fed, $p=0.3729 . \mathbf{g}$ VC treated: normal vs cuprizone-fed, $p=0.6316$. h P2C treated: normal vs cuprizone-fed, $p>0.9999$. $\mathbf{j}$ VC treated: normal vs cuprizone-fed, $p=0.6881$. P2C treated: normal vs cuprizone-fed, $p=0.1123$. Cuprizone-fed: VC vs P2C, $p=0.9999$

is not mediated through direct effects on OPC numbers or on their maturation into OLs.

\section{TLR2 tolerance decreases iNOS ${ }^{+}$microglia and increases}

\section{Arg $1^{+}$microglia following cuprizone-induced}

\section{demyelination}

Microglia represent an important regulator of CNS myelination and given their well-documented expression of TLR2 we next investigated their relevance in TLR2 tolerance-induced enhancement of remyelination [21-23]. We postulated that TLR2 tolerance induction would result in an enhancement of the microglial non-inflammatory/ pro-repair repair Arg1 $1^{+}$phenotype, known to be associated with enhanced remyelination [24-26].
To investigate this possibility, after the 2-week recovery period, CC sections were stained with antibodies to IBA1 to label all microglia, antibodies to iNOS to label microglia with a pro-inflammatory phenotype, and antibodies to Arg1 to label microglia with a non-inflammatory/pro-repair phenotype, markers known to correlate with pro- and anti-inflammatory functions, respectively, in the CNS [26-28]. Representative stains are shown in Fig. 4a-d. Sections were evaluated for the percentage of $\mathrm{IBA}^{+}$ microglia which stained positive for either iNOS or Arg1 alone ("single positives"), as well as the percentage of microglia staining with both markers ("double positives"). The percentages of single iNOS- or Arg1-positive microglia were each added to the percentages of double-positive 


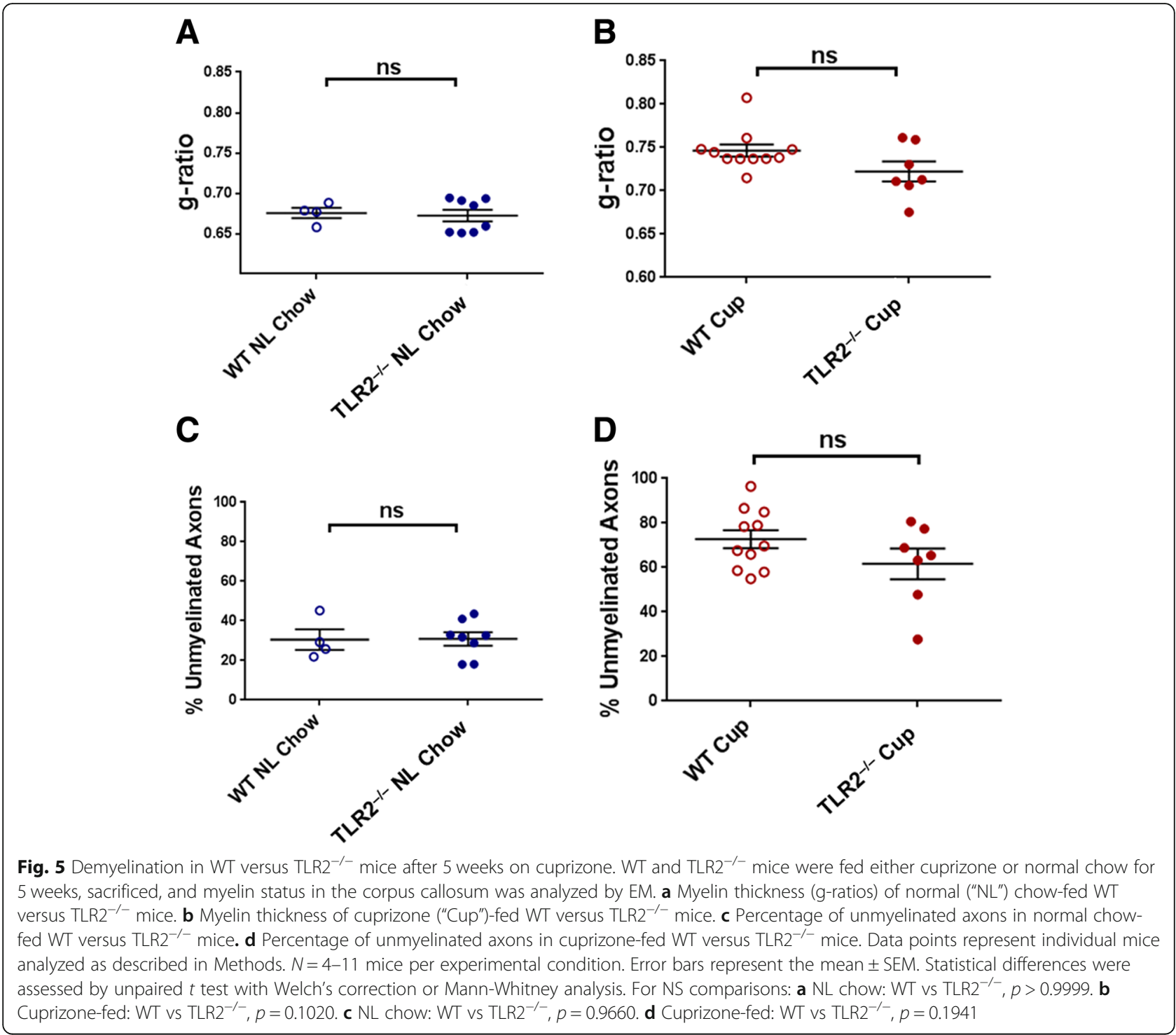

microglia to derive "total" iNOS- or "total" Arg1-positive microglia.

Cuprizone-fed mice demonstrated a significant microgliosis when compared to mice fed only with normal chow, and the increased number of microglia per square millimeter was the same regardless of $\mathrm{VC}$ or P2C treatment (Fig. 4e). Analysis of phenotypic staining revealed mice that were cuprizone-fed and treated with $\mathrm{VC}$ during the recovery period exhibited a significantly higher percentage of both singlepositive and total $\mathrm{iNOS}^{+}$microglia than VC-treated mice fed only with normal chow (Fig. 4h, f). These results suggest that cuprizone feeding induces an inflammatory phenotype in CC microglia. In contrast, mice that were cuprizone-fed but treated with P2C did not demonstrate this enhancement in the percentage of either single-positive or total $\mathrm{iNOS}^{+}$microglia
(Fig. 4h, f). This suggests that P2C treatment/TLR2 tolerance induction mitigated this cuprizone-induced inflammatory microglial phenotype.

Next, the percentage of $\operatorname{Arg} 1^{+}$microglia was analyzed in each cohort. In contrast to the increased percentage of $\mathrm{iNOS}^{+}$microglia in cuprizone-fed VCtreated mice, such mice exhibited a significant decrease in the percentage of single-positive $\operatorname{Arg} 1^{+}$ microglia when compared to normal chow-fed VCtreated mice (Fig. 4i). A similar decrease in the percentage of total $\mathrm{Arg}^{+}$microglia was also noted among cuprizone-fed VC-treated mice, but did not reach statistical significance (Fig. 4g). These results suggest that cuprizone feeding not only enhances the percentages of pro-inflammatory iNOS ${ }^{+}$microglia but also decreases the percentages of non-inflammatory/pro-repair repair $\operatorname{Arg} 1^{+}$microglia. 

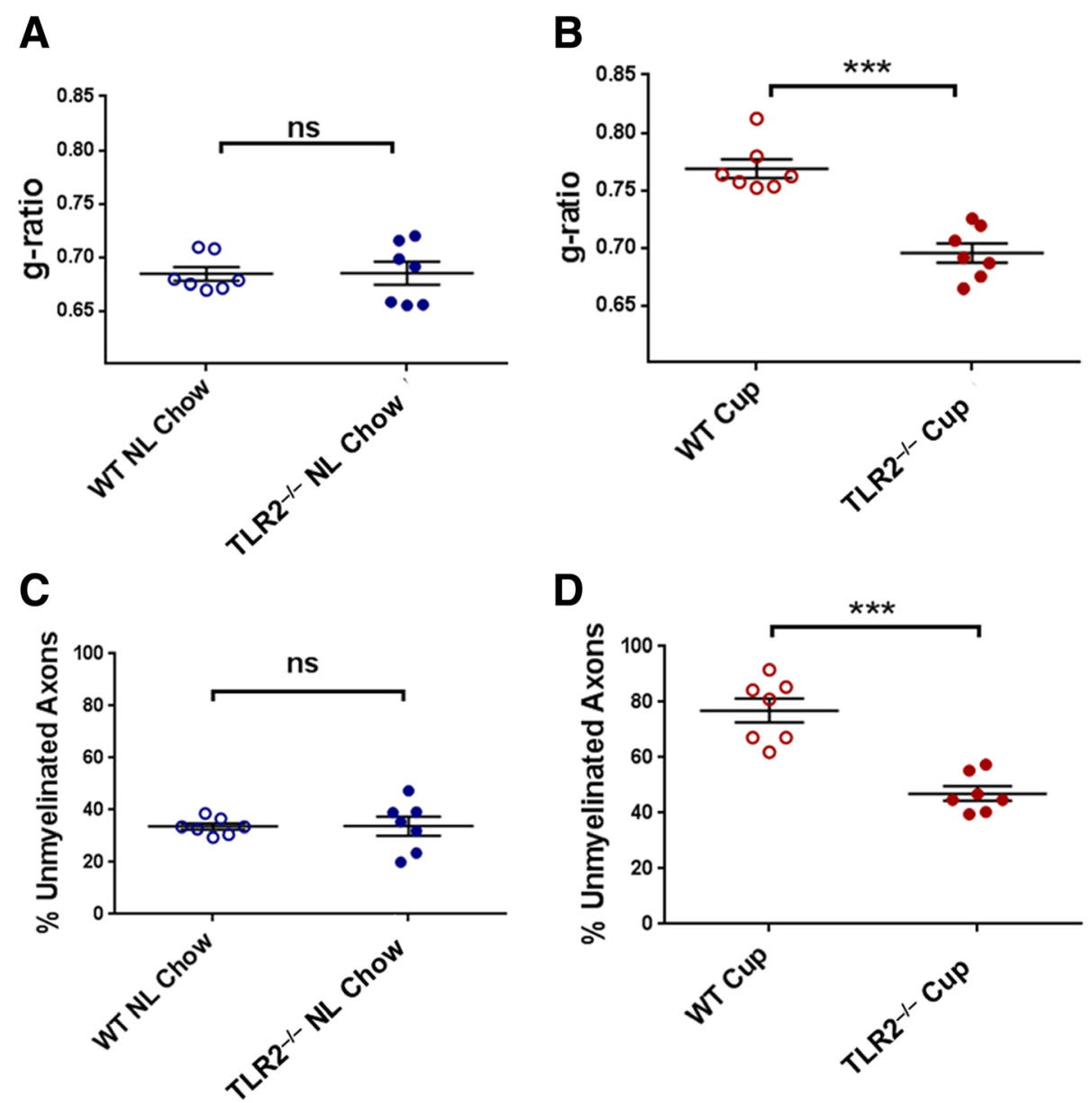

Fig. 6 Demyelination in WT versus TLR2 ${ }^{-/-}$mice after 5 weeks on cuprizone and 2 weeks on normal chow. WT and TLR2 ${ }^{-/-}$mice were fed either cuprizone or normal chow for 5 weeks, then all mice were fed normal chow for 2 weeks ("recovery period"). At the end of the 2-week recovery period, myelin status in the corpus callosum was analyzed by EM. a Myelin thickness (g-ratios) of normal ("NL") chow-fed WT versus TLR2 ${ }^{-1-}$ mice. b Myelin thickness of cuprizone ("Cup")-fed WT versus TLR2 ${ }^{-1-}$ mice. c Percentage of unmyelinated axons in normal chow-fed WT versus TLR2 ${ }^{-1-}$ mice. $\mathbf{d}$ Percentage of unmyelinated axons in cuprizone-fed WT versus TLR2 ${ }^{-/-}$mice. Data points represent individual mice analyzed as described in the "Methods" section. $N=7$ mice per experimental condition. Error bars represent the mean \pm SEM. Statistical differences were assessed by Mann-Whitney analysis. ${ }^{* * *} p<0.001$. For NS comparisons: a NL chow: WT vs TLR2 ${ }^{-1-}, p=0.9656$. c NL chow: WT vs TLR2 ${ }^{-1-}, p=0.9636$

A strikingly opposite trend was found among cuprizonefed, P2C-treated mice. Rather than a significant decrease in the percentage of $\operatorname{Arg} 1^{+}$microglia, the cuprizone-fed, P2Ctreated/TLR2-tolerized mice demonstrated a significantly higher percentage of both single-positive and total Arg1 ${ }^{+}$ microglia (Fig. 4i, g, j). The iNOS ${ }^{+} \mathrm{Arg}^{+}{ }^{+}$double-positive microglia were not significantly different across groups (Fig. 4j), indicating that the switch from single positive iNOS ${ }^{+}$-polarized to single positive $\mathrm{Arg} 1^{+}$-polarized microglia was the primary driver behind this phenotypic change. In sum, the microglial analyses indicate that TLR2 tolerance induction during the postcuprizone recovery phase results in a major microglial phenotypic switch, with a significant decrease in the percentage of iNOS $^{+}$pro-inflammatory microglia and a simultaneous increase in the percentage of $\operatorname{Arg} 1^{+}$ non-inflammatory microglia.

\section{$\mathrm{TLR2}^{-/-}$mice exhibit enhanced remyelination after} cuprizone feeding

To confirm that the enhanced remyelination in P2Ctreated mice was a result of TLR2 tolerance induction and not related to repeated low dose TLR2 stimulation, remyelination studies were performed utilizing TLR2deficient (TLR2 ${ }^{-1-}$ ) mice. Wild-type (WT; C57Bl/6) and $\mathrm{TLR}^{-/-}$mice (C57Bl/6 background) were fed for 5 weeks with either normal chow or cuprizone and then given a 2-week period on normal chow to allow for remyelination. In these studies, mice were not treated with either $\mathrm{VC}$ or $\mathrm{P} 2 \mathrm{C}$ during the 2-week recovery period (Fig. 1b).

To determine if WT and TLR2 ${ }^{-1-}$ mice differ in the degree of baseline demyelination mediated by 5 weeks of cuprizone exposure, normal chow-fed and cuprizone-fed mice were analyzed immediately after the initial 5-week 


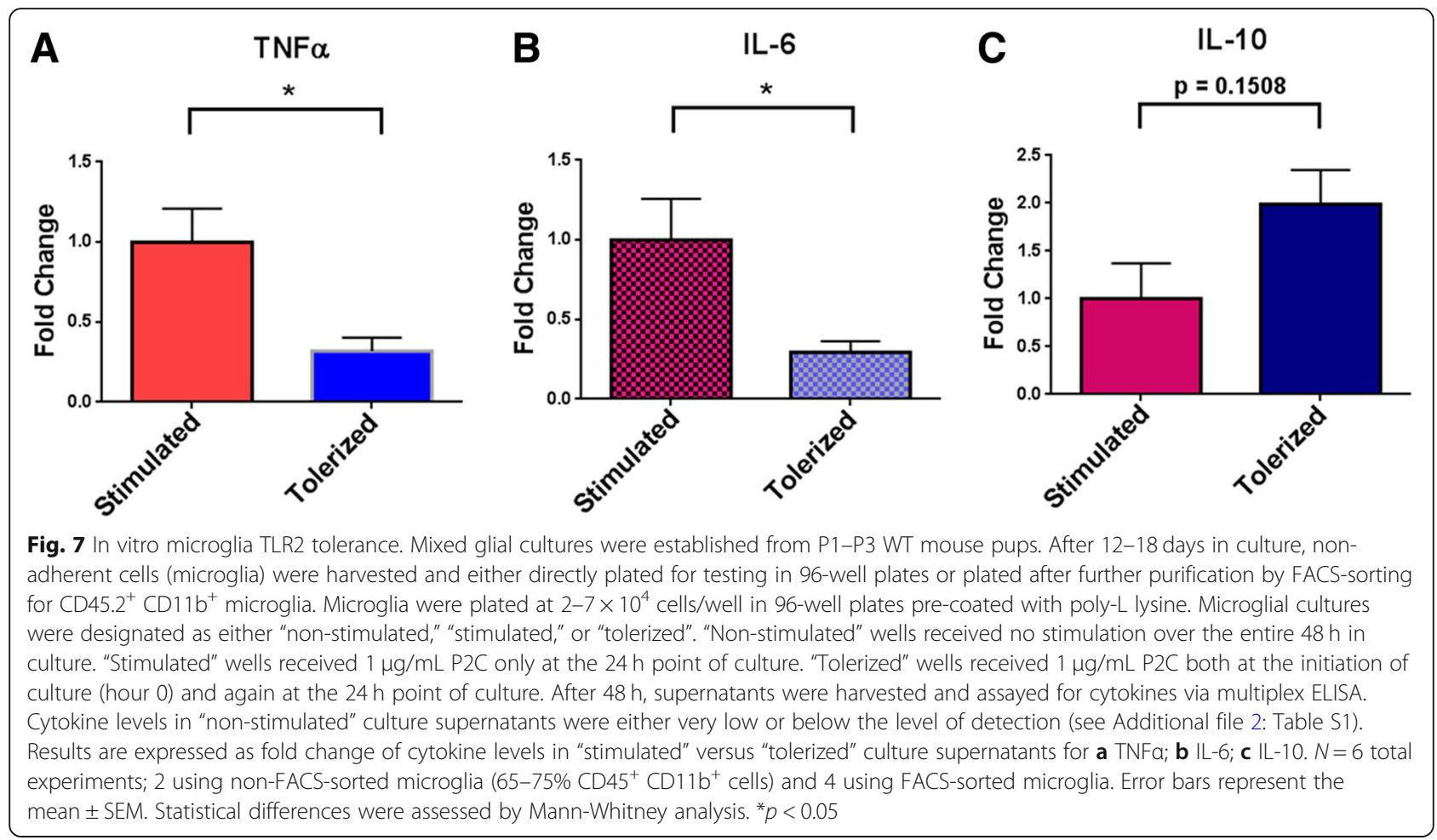

demyelination period (Fig. 1b). EM analysis confirmed that neither unmyelinated axon frequency nor myelin thickness differed between WT and TLR2 ${ }^{-1-}$ mice fed normal chow for 5 weeks (Fig. 5a, c). Importantly, these parameters also did not differ between WT and TLR2 $2^{-1-}$ mice fed cuprizone for 5 weeks (Fig. 5b, d). Thus, $\mathrm{TLR}^{-/-}$mice were not more or less susceptible than WT mice to cuprizone-mediated demyelination, consistent with previous reports [13].

Next, WT and TLR2 ${ }^{-1-}$ mice were analyzed after 5 weeks of cuprizone or normal chow followed by a 2week recovery period on normal chow. Similarly, myelin parameters did not differ between WT and TLR2 ${ }^{-/-}$ mice fed normal chow for the entire 5 and 2 week periods (Fig. 6a, c). In contrast, after cuprizone feeding and a 2-week recovery period on normal chow, TLR2 ${ }^{-/-}$ mice exhibited significantly enhanced recovery of myelinated axon frequency and myelin thickness when compared to WT mice (Fig. 6b, d). These results are consistent with earlier findings [15], suggesting that endogenous TLR2 signaling inhibits myelin repair. Moreover, these results are consistent with our conclusion that enhanced myelin repair following repeated low-dose P2C treatment is mediated by TLR2 tolerance induction rather than repeated TLR2 stimulation.

\section{In vitro TLR2 tolerance induction in microglia}

Previous studies reported in vitro induction of TLR2 tolerance in bone marrow-derived macrophages (BMDMs), showing that TLR tolerance can be observed in vitro even after a single round of TLR stimulation $[10,20]$. To further examine the effect of TLR2 tolerance on microglia, cultures of primary microglia were derived and tolerized in vitro using P2C. Primary microglia were isolated from cultures of whole mouse pup brains and, in some experiments, were FACS-sorted for purity. The isolated microglia were re-cultured in 96-well plates and remained either non-stimulated for $48 \mathrm{~h}$, stimulated by the addition of $1 \mu \mathrm{g} / \mathrm{mL} \mathrm{P2C}$ at $24 \mathrm{~h}$ of culture ("stimulated protocol"), or stimulated by the addition of $1 \mu \mathrm{g} /$ $\mathrm{mL}$ P2C at both the initiation of culture (hour 0 ) and 24 $\mathrm{h}$ of culture ("tolerance protocol"). After $48 \mathrm{~h}$, culture supernatants were harvested and assayed for levels of tumor necrosis factor $\alpha$ (TNF $\alpha$ ), interleukin 6 (IL-6), and interleukin 10 (IL-10) by ELISA.

Two studies were performed in which microglia were not purified by FACS-sorting. In these studies, the purity of the isolated microglia $\left(\mathrm{CD} 45^{+} \mathrm{CD} 11 \mathrm{~b}^{+}\right)$was $65-75 \%$. Four studies were performed in which microglia were isolated from cultures and then FACs-sorted. In these, the microglia purity was $>99 \%$ (Additional file 1: Fig. S1). The results of the $48 \mathrm{~h}$ testing of microglial stimulation and tolerance did not differ significantly between studies that used non-sorted versus sorted microglia, and therefore, the results of all six studies are compiled together in Fig. 7.

Cultures of microglia that received no in vitro stimulation with $\mathrm{P} 2 \mathrm{C}$ produced very low levels of cytokines. Microglial cultures stimulated with P2C only at $24 \mathrm{~h}$ 
("stimulated protocol") produced significant amounts of TNF $\alpha$ and IL-6. In contrast, microglia cultures stimulated with P2C at both 0 and $24 \mathrm{~h}$ ("tolerance protocol") produced significantly less TNF $\alpha$ and IL-6 (Fig. 7a, b; Additional file 1: Fig. S1). These results indicate that microglia, as with BMDM, can be induced to become TLR2 tolerant. While TLR2 tolerance induction resulted in decreased production of the pro-inflammatory cytokines TNF $\alpha$ and IL-6, it had the opposite effect on the production of the regulatory cytokine IL-10. Although not quite reaching statistical significance, microglia stimulated with $\mathrm{P} 2 \mathrm{C}$ at both 0 and $24 \mathrm{~h}$ ("tolerance protocol") produced nearly twofold more IL-10 when compared with microglia stimulated only at $24 \mathrm{~h}$ ("stimulated protocol") (Fig. 7c). Enhanced expression of IL-10 as a result of TLR tolerance induction has previously been documented in dendritic cells [29]. Overall, these in vitro results are consistent with our systemic in vivo TLR2 tolerance results described above in terms of TNF $\alpha$ production, confirming that isolated microglia manifest a less pro-inflammatory and more regulatory function when TLR2 tolerance is induced.

\section{Discussion}

A growing body of evidence implicates TLR2 signaling as a key mechanistic factor in the pathogenesis of MS and EAE [10-12], and the potential role of the microbiome in the pathogenesis of MS further complicates the issue. We have previously reported that a microbiomederived bacterial lipopeptide, Lipid 654, can be detected in the serum of all healthy individuals but is found at significantly lower levels in the serum of MS patients [16]. Based on this finding, we postulated that a normal function of the microbiome is to chronically expose the systemic innate immune system to low levels of microbial products which then serve to tolerize (i.e., regulate) innate responses. The homeostatic level of microbiomemediated tolerance normally achieved is appropriate for responding to infectious agents without over-responding to endogenous TLR-signaling agonists (e.g., DAMPs), the latter potentially resulting in autoimmunity.

The concept of TLR tolerance induction is well established both in vitro and in vivo [20]. This phenomenon involves initial ligation and signaling through a TLR followed by significantly diminished responses upon subsequent stimulation through the same TLR or other related TLRs. This mechanism of tolerance has been shown to be mediated by the upregulation of miRNAs that mediate cytokine production [30], increased expression of the NFKB regulator A20 [31], and activation of intracellular regulatory molecules including IRAK-M that effectively diminish subsequent ligand-induced responses [20]. We have postulated that when microbiome-derived products (e.g., Lipid 654) do not access the systemic circulation in concentrations that are sufficient to induce an appropriate level of tolerance, the normal regulatory function of the microbiome is lost and innate immune responses are over-responsive and potentially self-reactive. The significantly lower level of serum Lipid 654 that we reported in MS patients suggested that the "normal" level of microbiome-induced tolerance and regulation of innate immune responsiveness is deficient in MS.

In proof of concept studies, we reported that repeated daily administration of low dose, non-inflammatory levels of a TLR2 ligand induced a systemic reduction in TLR2 signaling ("TLR2 tolerance") and attenuated disease in a T cell transfer model of EAE [10]. In this study, TLR2 tolerance induction was associated with deficient activation of CNS antigen-presenting cells and significantly diminished CNS inflammation [10]. In a subsequent proof of concept study, we found that $50 \%$ of MS patients exhibited enhanced peripheral blood monocyte TLR2-stimulated responses, further suggesting deficient regulation of TLR2 responses in these patients [17]. These studies add confirmation to the underlying postulate that lack of sufficient circulating levels of microbiome-derived microbial products in MS results in defective systemic innate immune regulation and TLR2 over-responsiveness.

MS not only involves an autoimmune CNS inflammatory process, but also involves a defect in the ability to remyelinate the neurons demyelinated in this inflammatory process $[3,8,9]$. In addition to the controversial role of TLR2 in the inflammatory pathogenesis of MS and EAE, a small number of murine studies have also suggested that TLR2 signaling can inhibit remyelination. Prior studies reported that the in vitro maturation of OPC to OL is inhibited by TLR2 ligation and that hyaluronate, an endogenous TLR2 ligand (i.e., a DAMP), may be a relevant mediator of this inhibition of remyelination [15]. Furthermore, mice with a global deletion in TLR2 demonstrated enhanced remyelination in vivo after direct CNS administration of lysolecithin to induce demyelination [15], although these studies did not examine cuprizone-induced demyelination. Additional evidence suggesting an inhibitory role for TLR2 signaling in CNS remyelination include a study demonstrating that the TLR2 agonist, P3C, inhibited the promyelinating effects of erythropoietin [14], and a study reporting that cuprizone feeding resulted in increased expression of TLR2 in the CC and hippocampus [13]. Based on these prior studies of the potential involvement of TLR2 in the remyelination process, the lack of feasibility of a practical therapeutic approach for deleting TLR2 in vivo, and our EAE study suggesting the potential for a therapeutic reduction in TLR2 signaling using TLR2 tolerance induction [10], the goal in the present 
study was to test the role of TLR2 tolerance induction in the process of remyelination.

In the present study, mice were fed with cuprizone for 5 weeks to induce a non-inflammatory demyelination, followed by a 2-week feeding with normal chow to allow for remyelination. We asked whether diminishing TLR2 signaling by inducing systemic TLR2 tolerance during the 2-week recovery period would enhance remyelination. Our results showed that inducing TLR2 tolerance during the recovery period resulted in a significant enhancement in remyelination as documented by EM evaluation of both percentages of unmyelinated axons and myelin thickness (g-ratios).

We chose to focus on the 2-week post-cuprizone feeding time point to evaluate the effects of TLR2 tolerance induction on remyelination based on the well-documented time course of cuprizone-induced demyelination and recovery. We determined that 2 weeks post-cuprizone-cessation would provide the optimal window for assessing potential TLR2 toleranceinduced effects on remyelination. Numerous published studies have investigated the kinetics of remyelination after the cessation of cuprizone [32-34]. Importantly, these studies documented that complete remyelination, i.e., remyelination approaching control levels, is normally not seen for at least $4-5$ weeks after cuprizone is stopped. In contrast, our results demonstrate that at the 2-week post-cuprizone time point, TLR2tolerant mice demonstrate myelin metrics that are already statistically identical to control cohorts, i.e., mice that have never been exposed to cuprizone.

Using immunohistofluorescent approaches, we found that the TLR2 tolerance-induced enhancement in remyelination was not associated with changes in the number of OPCs or mature OLs, but rather was associated with a change in the balance of microglial phenotypes from pro-inflammatory iNOS ${ }^{+}$to a non-inflammatory/pro-repair $A r g 1^{+}$phenotype. Functionally, microglia (like macrophages) can be divided into different states of activation. M1 microglia are described as "pro-inflammatory" with regards to their cytokine secretion while M2 microglia ("alternatively activated" or "anti-inflammatory" microglia) are associated with extracellular matrix remodeling, progenitor cell differentiation, and tissue regeneration [24, 25, 35]. It has become clear that the M1/ M2 paradigm is too simplified to describe the complexity of microglia in vivo. The terms pro-inflammatory and anti-inflammatory more accurately describe the significant in vivo functional differences associated with iNOS ${ }^{+}$ Arg1 ${ }^{-}$versus iNOS $^{-} \mathrm{Arg}^{+}$microglia.

One limitation of our finding is that we have based our in vivo microglial phenotypic characterization on iNOS versus Arg1 expression rather than a more broad characterization at the transcriptome level. However, it should be noted that important aspects of macrophage and microglial biology are fundamentally driven by the phenotype of arginine metabolism utilized and, consistent with the functional in vivo differences in these microglia, pro-inflammatory and anti-inflammatory/prorepair cells metabolize arginine differently [36]. Proinflammatory cells utilize the enzyme nitric oxide synthase to metabolize arginine to nitric oxide and citrulline while anti-inflammatory/pro-repair cells utilize arginase to hydrolyze arginine to ornithine and urea. Importantly, both of these arginine metabolic pathways cross-inhibit each other [36].

Our results suggest that the enhanced remyelination seen with TLR2 tolerance is mediated, at least in part, by effects on microglial phenotype, and specifically on an increase in the percentage of $\mathrm{iNOS}^{-} \mathrm{Arg} 1^{+}$microglia. "M2" polarization of microglia has been documented to result in enhanced remyelination, and while there is not yet a clear understanding of how M2 microglia mediate enhanced remyelination, proposed mechanisms include increasing OPC to OL differentiation $[26,37]$ and increasing phagocytic clearance of myelin debris [38, 39]. In addition to our in vivo analysis, we also used an in vitro approach, confirming that microglia are easily induced to become TLR2 tolerant following a single stimulation with a TLR2 agonist. Moreover, in vitro TLR2-tolerized microglia demonstrated reduced secretion of pro-inflammatory cytokines and increased the production of the immune-regulatory cytokine IL-10. These results are in agreement with our previous in vitro studies of murine BMDMs [10] and in agreement with studies using primary rat microglial cultures induced to TLR4 tolerance using LPS [40].

Finally, with the goal of confirming that the enhanced remyelination documented in $\mathrm{P} 2 \mathrm{C}$-treated mice was a result of TLR2 tolerance induction and not related to repeated low dose TLR2 stimulation, we performed remyelination studies utilizing TLR2 ${ }^{-1}$ mice. In confirmation of our TLR2 tolerance results, we documented that $\mathrm{TLR}^{-/-}$mice show a significant enhancement in remyelination during recovery from cuprizone exposure. Studying mice with a global deletion of TLR2 precludes our commenting on the mechanistic role of microglia in the enhanced remyelination observed in these mice. To this end, future studies will utilize mice with microglialspecific deletion of TLR2.

In addition to $\mathrm{OPC}, \mathrm{OL}$, and microglia, astrocytes have been described as having both an enhancing and inhibitory effect on remyelination. As reviewed by Alizadeh et al. [41], astrocytes have been documented to play a role in clearance of myelin debris, modulating OL activity through intercellular connections, production of OL growth factors, and secreting chemokines that recruit cells, including microglia, into sites of demyelination. 
However, astrocytes also form glial scars which play an inhibitory role in CNS remyelination in part through the secretion of chondroitin sulfate proteoglycans (CSPGs) and other moieties which limit the ability of OPCS to migrate and differentiate into mature myelinating OLS [41]. In the present study, we have not specifically examined the effects of TLR2 tolerance induction on astrocytes but future studies will not only focus on mice with microglial-specific TLR2 deletion, but also on mice with astrocytic-specific deletion of TLR2.

The present study represents the next phase of proof-of-concept studies in which we investigate the use of TLR2 tolerance induction as a therapeutic approach in MS and potentially in other neuroinflammatory diseases as well. Unlike total genetic deletion of TLR2 (which would not be feasible in humans), the induction of TLR2 tolerance allows for regulation of the level of TLR2 responsiveness. Thus, our approach does not completely eliminate TLR2 signaling (e.g., see Fig. 1c), but rather "rheostats" it down to a level that we believe is significantly less pathogenic when receiving the relatively weak signals from endogenous ligands such as DAMPs. Importantly, in regard to the feasibility of applying this approach in humans, using careful titration, an appropriately low dose of TLR2 ligand(s) could be chosen that would induce tolerance without inducing pro-inflammatory signs or symptoms. This is the approach we have used to determine the TLR2 tolerizing dose of P2C administered in our studies. In mice, after the administration of the first low dose of $\mathrm{P} 2 \mathrm{C}$, which results most often in either no clinical effect or, at most, in a very mild hunching posture, all subsequent doses of P2C (with tolerance now induced) elicit no clinical signs. A similar approach is theoretically feasible in humans as well. Thus, we believe that "TLR2 tolerant" individuals will manifest no signs of proinflammatory effects from the low dose administration of ligands, but importantly will demonstrate a level of systemic TLR responsiveness that is diminished from pre-tolerance levels, while still allowing for appropriate responsiveness to the strong ligand signals presented by infectious organisms.

\section{Conclusion}

The present study presents evidence that the innate immune system, and TLR2 specifically, has a role in the process of remyelination. Moreover, together with our previous findings of lower serum Lipid 654 levels in MS patients [16], TLR2 tolerance-induced attenuation of EAE [10], and enhanced TLR2 responsiveness among MS patients [17], the present results represent further proof-of-concept evidence that inducing TLR2 tolerance in vivo mediates functional changes in TLR2 signaling that has physiologic and disease-relevant effects. By demonstrating the relevance of TLR2 signaling in remyelination, the present study also confirms earlier studies suggesting that TLR2 signaling plays a critical role in the defect in remyelination noted in patients with MS. Collectively, the present results and our previous EAE study suggest that induction of TLR2 tolerance may represent a novel two-pronged approach to MS treatment, inhibiting autoimmune inflammation while simultaneously facilitating myelin repair.

\section{Additional files}

Additional file 1: Figure S1. Microglia FACS-sorting strategy. Nonadherent cells (microglia) from 12 to 18 day mixed glial cultures were harvested, antibody stained, and purified by FACS-sorting for CD45.2 $2^{+}$

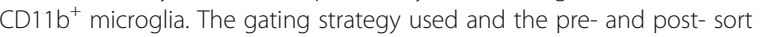
outcomes are depicted. (JPG $205 \mathrm{~kb}$ )

Additional file 2: Table S1. Cytokine levels in microglial culture supernatants. 96-well microglial cultures were designated as either "nonstimulated", "stimulated" or "tolerized". "Non-stimulated" wells received no stimulation over the entire 2 days of culture. "Stimulated" wells received $1 \mu \mathrm{g} / \mathrm{mL}$ P2C at the 24 hour time point. "Tolerized" wells received $1 \mu \mathrm{g} / \mathrm{mL}$ P2C both at the initiation of culture and at the 24 hour time point. After 48 hours, supernatants were harvested and assayed for cytokines via multiplex enzyme-linked immunosorbent assay (ELISA). Mean values are calculated from 2 replicate ELISA determinations and represent pg/ml. (JPG 463 kb)

\section{Abbreviations}

ANOVA: Analysis of variance; Arg1: Arginase 1; CC: Corpus callosum; CC1: Mouse monoclonal antibody marketed as anti-adenomatous polyposis coli clone CC1; CD: Cluster of differentiation; CNS: Central nervous system; DAPI: 4',6-diamidino-2-phenylindole; EAE: Experimental autoimmune encephalomyelitis; ELISA: Enzyme-linked immunosorbent assay; EM: Electron microscopy; i.v.: Intravenous; IBA1: Ionized calcium binding adaptor molecule 1; IL-10: Interleukin 10; IL-6: Interleukin 6; iNOS: Inducible nitric oxide synthase; MS: Multiple sclerosis; ns: Not significant; OL: Oligodendrocyte; OPC: Oligodendrocyte precursor cell; P2C: Pam2CSK, a synthetic diacylated lipopeptide; P3C: Pam3CSK , a synthetic triacylated lipopeptide;

PBS: Phosphate-buffered saline; PDGFRa: Platelet-derived growth factor receptor a; PFA: Paraformaldehyde; RRMS: Relapsing-remitting multiple sclerosis; SPMS: Secondary progressive multiple sclerosis; TEM: Transition electron microscope; TLR2: Toll-like receptor 2; TLR2-/- : Toll-like receptor 2deficient; TNFa: Tumor necrosis factor a; VC: Vehicle control; WT: Wild-type

\section{Acknowledgements \\ The authors would like to thank Dr. Glenn Matsushima, University of North Carolina School of Medicine, for his expert advice on using the cuprizone model, and Ms. Maya Yankova of the UConn Health Electron Microscopy Core and Dr. Evan Jellison of the UConn Health Flow Cytometry Core for their expert technical assistance.}

\section{Authors' contributions}

RC and NW planned and organized all experiments. NW conducted all cuprizone studies and resulting electron microscopy data collection, statistical analysis, and interpretation. Immunofluorescence imaging, statistical analysis, and interpretation were performed by NW, with technical instruction and assistance from DP, SY, KK, and JP. MK performed in vitro microglia assays with technical instruction and assistance from AN and SC. NW drafted the

manuscript with discussions and contributions from FN, JP, and RC throughout the process. All authors read and approved the final manuscript.

\section{Funding}

This work was supported by Connecticut Bioscience Innovation Fund Grant \# 517 and University of Connecticut Health Center Grant \# G401691. 


\section{Availability of data and materials}

The datasets used and/or analyzed during the current study are available from the corresponding author on reasonable request. The g-ratio analysis plug-in and source code for the ImageJ software (http://rsbweb.nih.gov/ij), which allowed for semi-automated analysis of randomly selected sets of fibers, are available online (http://gratio.efil.de).

\section{Ethics approval and consent to participate}

All mice were maintained under specific pathogen-free conditions in accordance with the guidelines for the Center for Comparative Medicine at UConn Health. All procedures were performed in compliance with Institutional Animal Care and Use Committee-approved protocols.

\section{Consent for publication}

Not applicable.

\section{Competing interests}

The authors declare that they have no competing interests.

\section{Author details}

'Department of Immunology, UConn Health, Farmington, CT 06030, USA. 2Department of Medicine, UConn Health, Farmington, CT 06030, USA. ${ }^{3}$ Department of Neuroscience, UConn Health, Farmington, CT 06030, USA ${ }^{4}$ Department of Periodontology, UConn Health, Farmington, CT 06030, USA. ${ }^{5}$ Present Address: Department of Microbiology, Perlmutter Cancer Center at NYU Langone School of Medicine, New York, NY 10016, USA.

\section{Received: 4 March 2019 Accepted: 9 July 2019}

\section{Published online: 27 July 2019}

\section{References}

1. Yong $\mathrm{H}$, Chartier $\mathrm{G}$, Quandt J. Modulating inflammation and neuroprotection in multiple sclerosis. J Neurosci Res. 2018;96(6):927-50.

2. Ransohoff RM, Hafler DA, Lucchinetti CF. Multiple sclerosis-a quiet revolution. Nat Rev Neurol. 2015;11(3):134-42.

3. Hanafy KA, Sloane JA. Regulation of remyelination in multiple sclerosis. FEBS Lett. 2011;585(23):3821-8.

4. Shirani A, Okuda DT, Stuve O. Therapeutic advances and future prospects in progressive forms of multiple sclerosis. Neurotherapeutics. 2016;13(1):58-69.

5. McTigue DM, Tripathi RB. The life, death, and replacement of oligodendrocytes in the adult CNS. J Neurochem. 2008;107(1):1-19.

6. Franklin RJ, Ffrench-Constant C. Remyelination in the CNS: from biology to therapy. Nat Rev Neurosci. 2008:9(11):839-55.

7. Goldman SA, Nedergaard M, Windrem MS. Glial progenitor cell-based treatment and modeling of neurological disease. Science. 2012;338(6106): 491-5.

8. Kremer D, Aktas O, Hartung HP, Kury P. The complex world of oligodendroglial differentiation inhibitors. AnnNeurol. 2011;69(4):602-18.

9. Kuhlmann T, Miron V, Cui Q, Wegner C, Antel J, Bruck W. Differentiation block of oligodendroglial progenitor cells as a cause for remyelination failure in chronic multiple sclerosis. Brain. 2008;131(Pt 7):1749-58.

10. Anstadt EJ, Fujiwara M, Wasko N, Nichols F, Clark RB. TLR tolerance as a treatment for central nervous system autoimmunity. J Immunol. 2016;197(6): 2110-8

11. Kirby TO, Ochoa-Reparaz J. The gut microbiome in multiple sclerosis: a potential therapeutic avenue. Med Sci (Basel). 2018;6(3). https://doi.org/10.33 90/medsci6030069. Review. PMID:30149548

12. Wang Y, Telesford KM, Ochoa-Reparaz J, Haque-Begum S, Christy M, Kasper EJ, et al. An intestinal commensal symbiosis factor controls neuroinflammation via TLR2-mediated CD39 signalling. Nat Commun. 2014; 5:4432.

13. Esser S, Gopfrich L, Bihler K, Kress E, Nyamoya S, Tauber SC, et al. Toll-like receptor 2-mediated glial cell activation in a mouse model of Cuprizoneinduced demyelination. Mol Neurobiol. 2018;55(8):6237-49.

14. Gyetvai G, Roe C, Heikal L, Ghezzi P, Mengozzi M. Leukemia inhibitory factor inhibits erythropoietin-induced myelin gene expression in oligodendrocytes. Mol Med. 2018:24(1):51

15. Sloane JA, Batt C, Ma Y, Harris ZM, Trapp B, Vartanian T. Hyaluronan blocks oligodendrocyte progenitor maturation and remyelination through TLR2. Proc Natl Acad Sci U S A. 2010;107(25):11555-60.
16. Farrokhi V, Nemati $R$, Nichols FC, Yao X, Anstadt E, Fujiwara $M$, et al. Bacterial lipodipeptide, Lipid 654, is a microbiome-associated biomarker for multiple sclerosis. Clin Transl Immunology. 2013;2(11):e8.

17. Fujiwara M, Anstadt EJ, Flynn B, Morse K, Ng C, Paczkowski P, et al. Enhanced TLR2 responses in multiple sclerosis. Clin Exp Immunol. 2018; 193(3):313-26.

18. Goebbels S, Oltrogge JH, Kemper R, Heilmann I, Bormuth I, Wolfer S, et al. Elevated phosphatidylinositol 3,4,5-trisphosphate in glia triggers cell-autonomous membrane wrapping and myelination. J Neurosci. 2010;30(26):8953-64.

19. Roy J. Primary microglia isolation from mixed cell cultures of neonatal mouse brain tissue. Brain Res. 2018;1689:21-9.

20. Medvedev AE, Sabroe I, Hasday JD, Vogel SN. Tolerance to microbial TLR ligands: molecular mechanisms and relevance to disease. J Endotoxin Res. 2006:12(3):133-50

21. Chen K, Iribarren P, Hu J, Chen J, Gong W, Cho EH, et al. Activation of Toll-like receptor 2 on microglia promotes cell uptake of Alzheimer disease-associated amyloid beta peptide. J Biol Chem. 2006;281(6): 3651-9.

22. Kim D, Kim MA, Cho IH, Kim MS, Lee S, Jo EK, et al. A critical role of toll-like receptor 2 in nerve injury-induced spinal cord glial cell activation and pain hypersensitivity. J Biol Chem. 2007;282(20):14975-83.

23. Olson JK, Miller SD. Microglia initiate central nervous system innate and adaptive immune responses through multiple TLRs. J Immunol. 2004;173(6): 3916-24.

24. Cherry JD, Olschowka JA, O'Banion MK. Neuroinflammation and M2 microglia: the good, the bad, and the inflamed. J Neuroinflammation. 2014; 11.98

25. Nakagawa Y, Chiba K. Diversity and plasticity of microglial cells in psychiatric and neurological disorders. Pharmacol Ther. 2015:154:21-35.

26. Miron VE, Boyd A, Zhao JW, Yuen TJ, Ruckh JM, Shadrach JL, et al. M2 microglia and macrophages drive oligodendrocyte differentiation during CNS remyelination. Nat Neurosci. 2013;16(9):1211-8.

27. Zhou T, Huang Z, Zhu X, Sun X, Liu Y, Cheng B, et al. Alpha-1 antitrypsin attenuates M1 microglia-mediated neuroinflammation in retinal degeneration. Front Immunol. 2018:9:1202.

28. Han Z, Zhao H, Tao Z, Wang R, Fan Z, Luo Y, et al. TOPK promotes microglia/macrophage polarization towards M2 phenotype via inhibition of HDAC1 and HDAC2 activity after transient cerebral ischemia. Aging Dis. 2018;9(2):235-48.

29. Yanagawa Y, Onoe K. Enhanced IL-10 production by TLR4- and TLR2-primed dendritic cells upon TLR restimulation. J Immunol. 2007;178(10):6173-80.

30. Nahid MA, Benso LM, Shin JD, Mehmet H, Hicks A, Ramadas RA. TLR4, TLR7/ 8 agonist-induced miR-146a promotes macrophage tolerance to MyD88dependent TLR agonists. J Leukoc Biol. 2016;100(2):339-49.

31. Abbasi A, Forsberg K, Bischof F. The role of the ubiquitin-editing enzyme A20 in diseases of the central nervous system and other pathological processes. Front Mol Neurosci. 2015;8:21.

32. Hiremath MM, Chen VS, Suzuki K, Ting JP, Matsushima GK. MHC class II exacerbates demyelination in vivo independently of T cells. $J$ Neuroimmunol. 2008:203(1):23-32.

33. Matsushima GK, Morell P. The neurotoxicant, cuprizone, as a model to study demyelination and remyelination in the central nervous system. Brain Pathol. 2001;11(1):107-16.

34. Sachs HH, Bercury KK, Popescu DC, Narayanan SP, Macklin WB. A new model of cuprizone-mediated demyelination/remyelination. ASN Neuro. 2014;6(5). https://doi.org/10.1177/1759091414551955. 2014. PMID:25290063

35. Amici SA, Dong J, Guerau-de-Arellano M. Molecular mechanisms modulating the phenotype of macrophages and microglia. Front Immunol. 2017;8:1520

36. Rath M, Muller I, Kropf $\mathrm{P}$, Closs El, Munder M. Metabolism via arginase or nitric oxide synthase: two competing arginine pathways in macrophages. Front Immunol. 2014;5:532

37. Miron VE, Franklin RJ. Macrophages and CNS remyelination. J Neurochem. 2014;130(2):165-71.

38. Laflamme $N$, Cisbani $G$, Prefontaine $P$, Srour $Y$, Bernier J, St-Pierre MK, et al. mCSF-induced microglial activation prevents myelin loss and promotes its repair in a mouse model of multiple sclerosis. Front Cell Neurosci. 2018;12:178.

39. Lloyd AF, Davies CL, Miron VE. Microglia: origins, homeostasis, and roles in myelin repair. Curr Opin Neurobiol. 2017;47:113-20. 
40. Ajmone-Cat MA, Nicolini A, Minghetti L. Prolonged exposure of microglia to lipopolysaccharide modifies the intracellular signaling pathways and selectively promotes prostaglandin E2 synthesis. J Neurochem. 2003:87(5):1193-203.

41. Alizadeh A, Dyck SM, Karimi-Abdolrezaee S. Myelin damage and repair in pathologic CNS: challenges and prospects. Front Mol Neurosci. 2015;8:35.

\section{Publisher's Note}

Springer Nature remains neutral with regard to jurisdictional claims in published maps and institutional affiliations.

Ready to submit your research? Choose BMC and benefit from:

- fast, convenient online submission

- thorough peer review by experienced researchers in your field

- rapid publication on acceptance

- support for research data, including large and complex data types

- gold Open Access which fosters wider collaboration and increased citations

- maximum visibility for your research: over $100 \mathrm{M}$ website views per year

At BMC, research is always in progress.

Learn more biomedcentral.com/submissions 